\section{Very early recognition of coronary heart disease}

Cardiologists from many countries came together earlier this month at a London symposium on the very early recognition of coronary heart disease organised by the Cardiothoracic Institute and the National Heart Hospital-and at a preliminary "think-tank" meeting at Leeds Castle, Kent. They reached two important conclusions. Firstly, while we know that coronary heart disease shows itself by abnormalities in the pumping or electrical activity of the heart muscle (myocardial infarction, angina pectoris, heart failure, arrhythmias, and sudden death), we do not yet understand the precise relations between these end-stage events and coronary atherosclerosis and thrombosis, or the group-markers of risk such as cigarette smoking, raised serum lipid concentrations, and hypertension. While these interrelations remain enigmatic we cannot be confident that studies carried out on the heart, the coronary tree, or the blood will allow us to predict which individuals are destined to develop the disease. Secondly, the symposium questioned whether we are justified in expanding human and material resources in recognising any disease at an early stage unless detection can lead to useful action. Comparison with breast and lung cancer is appropriate, for in neither of these conditions is it self-evident that early detection necessarily helps patients.

Even though the meeting could not resolve these dilemmas it pointed to gaps in our knowledge and drew attention to technological advances in the non-invasive assessment of the heart and great vessels that should prove valuable in surveys of sizable groups. It highlighted the poor predictive value of risk markers (both simple ones such as family history, smoking habit, and weight, and resource-demanding investigations such as blood pressure, serum lipids, and electrocardiographic patterns at rest and in response to exercise). If we take 100 men with the three major risk markers (smoking, hypertension, and raised serum cholesterol) only eight develop clinical manifestations of coronary heart disease over the next 10 years, while 92 do not $^{1}$; conversely, most previously fit patients who developed coronary heart disease while under observation in the Seattle "heart-watch" programme had no conventional risk factors on entry. We must therefore realise that risk factors cannot be causal and that they have very poor predictive value. Furthermore, at present none of our more advanced tests (such as tests for thrombotic tendency or exercise stressing of the heart) improve the specificity and sensitivity of the predictions.

In contrast to the epidemiological gloom technological advances seemed to offer some hope of being able to define more accurately the relations between disease and symptoms (why do patients with coronary artery disease get chest pain? Is the pain chemically or mechanically mediated ?) and among the various disease elements (vessel wall and lumen disease, myocardial disease, electrical dysfunction). Our new-found ability to measure and display the heart wall and its movements by non-invasive methods such as echocardiography, electromagnetic field disturbances, and isotopic imaging adds another dimension to clinical investigation. A ciné-image may be built of the left ventricular cavity by gated isotope scanning after an intravenous injection, making it possible to calculate the ejection fraction and to identify dyskinetic segments in patients in whom conventional transarterial catheterisation of the left ventricle would be totally unjustified or harmful. Gamma-emitting isotope detection of areas of infarction is rapidly becoming more refined, and the use of positronemitting isotopes may soon enable us to detect myocardial lesions less than one centimetre in diameter.

The messages from the symposium are clear. Before more money is spent on screening for risk factors (as opposed to the good clinical practice of recording and modifying smoking habits, weight, and blood pressure) we need to improve the predictive power of our tests, and we need to decide how we are going to advise individuals found to be at risk. Moreover, though major advances may occur because a new technique becomes available, they are not inevitable. The new noninvasive imaging methods have great promise in research, but it is vital to ensure that their benefits to patients are commensurate with their cost to the community before putting them into service.

${ }^{1}$ Report of Intersociety Commission for Heart Disease Resources,
Circulation, 1970, 42A, 55.

\section{Research and cancer education}

The recent upsurge of interest in a preventive approach to the control of cancer has come at a time when finance for any new developments has become exceptionally hard to find. Fortunately, despite neglect by the NHS, there have been a few pioneer units examining the potential of both prevention and public education in this field, so that there is a body of practical experience on which to build. ${ }^{1}$

There are three separate tasks. Firstly, more needs to be done in primary prevention. While most cancer may prove to be due to environmental agents, ${ }^{2}$ for the time being tobacco and alcohol remain the only examples of substances in common use that have been proved to be major carcinogens. The campaigns so far mounted to discourage their consumption have been well intentioned rather than well researched. We need to know, for example, why the distribution of smokers in the British population has changed so dramatically in the last 20 years: $:^{3}$ the numbers of men in social class I who smoke have almost halved, yet their wives smoke as much as ever, and both men and women in social classes IV and $\mathrm{V}$ smoke more than they did in the 1950s.

Secondly, screening for presymptomatic disease needs critical examination. While there is evidence that the prognosis of cancer of the cervix is determined by its clinical stage at the time of presentation, there may not be the same clear-cut gain from other screening techniques. The results of mammographic screening ${ }^{4}$ have shown how difficult it may be to identify the population of women in whom early detection leads to a statistically significant improvement in cure of the disease.

Thirdly, far too many patients still present themselves for treatment with cancers that first gave them symptoms months if not years earlier. Education has a huge task ahead in trying to dispel the deep-rooted misconceptions that deter prompt action by individuals well aware of the nature of their symptoms. In Britain we have been far less open than the Americans about talking about cancer: few doctors here are prepared to tell a patient with a resectable tumour that it is malignant, so that we should not be surprised that many people still equate cancer with death.

If cancer education is to play a larger part in the NHS 
then we need to know that the advice given is sound and that it is effective in changing attitudes, in reducing delay in diagnosis, and-ultimately-in reducing mortality, and especially mortality in youth and middle age. The Cancer Research Campaign has recently allocated some of its funds to support research projects concerned with cancer education, so that finance is available for evaluation. Short of money, the NHS needs to make the best use of existing knowledge; and prevention offers the best hope of reducing both the toll of cancer and the demands it makes on resources.

1 Public Education about Cancer: Research Concepts and Theoretical Findings, UICC Monograph Series vol 5. Berlin, Springer-Verlag, 1967.

2 Doll, R, Fournal of the Royal College of Physicians, 1977, 2, 125.

3 Todd, G F, Social Class Variations in Cigarette Smoking and in Mortality from Associated Diseases. London, Tobacco Research Council, 1976.

4 Strax, P, Venet, L, and Shapiro, S, American fournal of Roentgenology, $1973,117,686$

5 Stark, A M, and Way, S, Cancer, 1974, 33, 1671.

\section{Congenital dislocation of the hip}

Growth and development of the hip joint depends upon congruous and stable articulation of its components if it is to proceed normally. If the joint is unstable-as occurs ${ }^{12}$ in three to six infants per 1000 live births-the abnormality must be recognised in neonatal life so that treatment can be given to prevent hip deformity.

The simple clinical methods of detection described by Ortolani $^{3}$ and Barlow ${ }^{4}$ have gained general acceptance, but despite this several hip dislocations still go unrecognised at birth. ${ }^{156}$ Poor technique may account for some errorsexamining a baby who is not relaxed or who is lying on a soft rather than a firm surface. If traction is applied to the limb rather than pressure on the thigh the hip may be fully abducted with an unstable hip reduced. ${ }^{6}$ But perhaps most important of all is failure to recognise limitation of abduction as a sign of congenital dislocation. ${ }^{2} 78$ In infants with frank dislocation, as opposed to subluxation or instability, the "clunk" sign may be absent, and the only abnormal finding may be that both hips show restriction of abduction in flexion.?

Diagnostic success depends on organisation-clearly easier to achieve in a large maternity unit than in a small peripheral one, or after domiciliary delivery. ${ }^{9}$ As $94 \%$ of births now take place in hospital in England and Wales ${ }^{10}$ the onus must be on the hospitals to provide this service. In a well-organised unit the missed dislocation rate may be as low as 1 in 8000 births. $^{9}$ Diagnostic accuracy of this order does, however, demand a high degree of perseverance and enthusiasm on the part of the examiner: it is unlikely if screening is left to junior staff whose posts are frequently changing.

In general, the outlook for infants diagnosed and treated from birth is excellent. In a long-term follow-up of 111 children treated early by splints Fredensborg" ${ }^{11}$ reported that all developed normal hips, apart from two who had minor deformities. Mitchell ${ }^{1}$ showed that $92 \%$ of abnormal hips developed satisfactorily with splinting from birth, and similar findings were reported by Williamson. ${ }^{6}$ Later diagnosis means more complex operations, and with such a high degree of success with relatively simple treatment in the neonatal period there is a natural tendency to overtreat. ${ }^{12}$ Nevertheless, though infrequent, the complications of splinting are serious.
Gore $^{13}$ described six patients who developed avascular necrosis of the opposite normal hip while being treated for unilateral dislocation by plaster casts; the dislocated hips also became avascular. The important safeguard is to avoid extremes of abduction. If there is some pelvic obliquity it may limit hip abduction and produce shortening of the leg, and in these cases splinting may lead to ischaemic necrosis of the femoral head, ${ }^{14}$ when simple abduction exercises would have been enough.

"Clicks" as opposed to the visible and audible Ortolani jerk or "clunk" are often a source of confusion. The former are usually of no importance, disappearing within a few weeks. For example, in one series of infants examined shortly after birth 138 were diagnosed as having subluxating hips. ${ }^{15}$ At a second examination at 7-10 days 62 were still abnormal, but by 3 weeks only three infants were considered to need treatment, and those were splinted. The high incidence of abnormality noted initially appeared to be due to the ease with which clicks may be elicited in the joints of the newborn. These infants do not require treatment, but they should be examined again at the age of 3 months and at 1 year.

The importance of early diagnosis cannot be overemphasised, but even with the greatest care the occasional unexplained failure to detect instability or dislocation will occur. ${ }^{156}$ To reduce to a minimum the delay before treatment starts ideally all children should be screened again during their first year.

${ }^{1}$ Mitchell, G P, fournal of Bone and foint Surgery, 1972, 54B, 4.

2 Paterson, D C, Clinical Orthopaedics and Related Research, 1976, 119, 28.

3 Ortolani, M, Pediatria (Naples), 1937, 45, 129.

${ }^{4}$ Barlow, T G, Fournal of Bone and foint Surgery, 1962, 44B, 292.

${ }^{5}$ Owen, R, Fournal of Bone and Foint Surgery, 1968, 50B, 453.

6 Williamson, J, fournal of Bone and foint Surgery, 1972, 54B, 13.

${ }^{7}$ Lloyd-Roberts, G C, and Swann, M, Fournal of Bone and foint Surgery, 1966, 48B, 666.

${ }^{8}$ Ortolani, M, Clinical Orthopaedics and Related Research, 1976, 119, 6.

${ }^{9}$ Mitchell, G P, fournal of the Royal College of Surgeons of Edinburgh, 1977, 22, 81.

${ }^{10}$ Registrar General's Stutistical Review of England and Wales for 1973, Tables, pt II Population. London, HMSO, 1975.

11 Fredensborg, N, fournal of Bone and foint Surgery, 1976, 58B, 272.

${ }^{12}$ Fredensborg, N, and Nilsson, B E. Clinical Orthopaedics and Related Research, 1976, 119, 89.

${ }^{13}$ Gore, D R, fournal of Bone and foint Surgery, 1974, 56A, 493.

${ }^{14}$ Lloyd-Roberts, G C, Orthopaedics in Infancy and Childhood, p 210. London, Butterworths, 1971.

${ }^{15}$ Nelson, M A, Fournal of Bone and foint Surgery, 1966, 48B, 388.

\section{Steroid-antibiotic combinations}

Ointments and creams containing a corticosteroid in combination with an antibiotic are prescribed widely by general practitioners and only slightly less often by most dermatologists. Many authors have, however, put forward objections to the practice, which have been more or less soundly supported by theoretical argument or by apparently reliable experimental evidence. It is accepted that there is no advantage in adding a corticosteroid to an antibiotic for treating a primary skin infection such as impetigo. On the other hand, a corticosteroidantibiotic combination is the treatment of choice in eczema of any type when there are clinical signs of bacterial infection. The controversy concerns those common cases in which eczema is not obviously infected: does the addition of an antibiotic or other antibacterial agent to a corticosteroid significantly accelerate the response to treatment? If it does, is the 